\title{
ROLE OF SEX HORMONES IN SEX CHANGE OF GROUPER
}

\section{Masaru Nakamura}

Sesoko Station, Tropical Biosphere Research Center, University of the Ryukyus, Sesoko 3422, Motobu, Okinawa 905-0227, Japan. Solution Oriented Research for Science and Technology (SORST), Japan Science and Technology Corporation, Saitama 32-0012, Japan., masaru@lab.u-ryukyu.ac.jp

Mohammad Ashraful Alam

Sesoko Station, Tropical Biosphere Research Center, University of the Ryukyus, Sesoko 3422, Motobu, Okinawa 905-0227, Japan.

Yasuhisa Kobayashi

Sesoko Station, Tropical Biosphere Research Center, University of the Ryukyus, Sesoko 3422, Motobu, Okinawa 905-0227, Japan.

Ramji Kumar Bhandari

Follow this and additional works at: https://jmstt.ntou.edu.tw/journal

Part of the Aquaculture and Fisheries Commons

\section{Recommended Citation}

Nakamura, Masaru; Alam, Mohammad Ashraful; Kobayashi, Yasuhisa; and Bhandari, Ramji Kumar (2007) "ROLE OF SEX HORMONES IN SEX CHANGE OF GROUPER," Journal of Marine Science and Technology. Vol. 15: Iss. 5, Article 3. DOI: $10.51400 / 2709-6998.2055$

Available at: https://jmstt.ntou.edu.tw/journal/vol15/iss5/3

This Research Article is brought to you for free and open access by Journal of Marine Science and Technology. It has been accepted for inclusion in Journal of Marine Science and Technology by an authorized editor of Journal of Marine Science and Technology. 


\section{ROLE OF SEX HORMONES IN SEX CHANGE OF GROUPER}

Acknowledgements

Laboratory of Reproductive Biology, National Institute for Basic Biology, Nishigonaka, Myodaiji, Okazaki, Aichi, Japan. 


\title{
ROLE OF SEX HORMONES IN SEX CHANGE OF GROUPER
}

\author{
Masaru Nakamura*,**, Mohammad Ashraful Alam*, Yasuhisa Kobayashi*, \\ Ramji Kumar Bhandari***
}

Key words: sex steroids, sex change, E. merra, protogynous grouper.

\begin{abstract}
Groupers are commercially important coral reef fishes because of their excellent flavor and high price. Due to their sex changing nature, commercial aquaculture operations have not yet been developed for groupers. Sex steroids play a critical role in the initiation of sex change, but their exact function and mechanism of action are unknown. We have investigated the role of sex steroids in protogynous sex change using the honeycomb grouper, Epinephelus merra, for a number of years. Our studies have shown that sex steroids play a major role in sex change. In this review, we integrate our findings to better understand the mechanism of sex change in groupers.
\end{abstract}

\section{INTRODUCTION}

Groupers of the genus Epinephelus are widely distributed throughout tropical and subtropical waters, and are very important aquaculture species. Most groupers are protogynous hermaphrodites, which change sex from female to male depending on their size and/or age [4], or on the basis of their complex social structure [12]. The process of sex change occurs in a number of ways, although it is always initiated and regulated by gonadal steroids [10]. For over 30 years, biologists have hypothesized about the involvement of steroid hormones in sex change [10], but the endocrine mechanisms of sex change are not clearly understood.

Our research focuses on analyzing the mechanism of protogynous sex change in groupers from an endocrinological viewpoint using morphological, physiological, and molecular techniques. We use the honeycomb

Author for Correspondence: M. Nakamura.

E-mail: masaru@lab.u-ryukyu.ac.jp.

*Sesoko Station, Tropical Biosphere Research Center, University of the Ryukyus, Sesoko 3422, Motobu, Okinawa 905-0227, Japan.

**Solution Oriented Research for Science and Technology (SORST), Japan Science and Technology Corporation, Saitama 32-0012, Japan.

*** Laboratory of Reproductive Biology, National Institute for Basic Biology, Nishigonaka, Myodaiji, Okazaki, Aichi, Japan. grouper, E. merra, as a model organism for protogynous sex change studies, because it is one of the smallest grouper species and is readily obtainable in the wild. Given our studies of the endocrine control of sex change in E. merra, the aim of this review is to describe the role of gonadal steroids and their sites of synthesis during the process of sex change. Additionally, we will focus on the manipulation of endocrine-controlling mechanisms to develop a method to initiate sex change of grouper in captivity.

\section{Changes of serum sex hormones during sex change}

We investigated changes in the gonadal structures and levels of serum sex steroid hormones during female- to-male sex change. On the basis of histological changes, entire process of sex change was assigned into four developmental phases: female, early transition (ET), late transition (LT), and male phase (Figure 1). At the female phase, the oocytes of several developmental stages were observed including gonial germ cells in the periphery of ovigerous lamellae (Figure 1a). At the beginning of ET phase, perinucleolar and previtellogenic oocytes began degenerating, followed by proliferation of spermatogonia toward the center of lamella (Figure $1 b)$. The LT phase was characterized by further degeneration of oocytes and rapid proliferation of spermatogenic germ cell throughout the gonad (Figure 1c). At the male phase, no ovarian cells were observed and testis had germ cells undergoing active spermatogenesis (Figure 1d). Serum levels of estradiol-17 $\beta$ (E2) were high in females in the breeding season, but low in the non-breeding female, transitional and male phase and those of 11-ketotestosterone (11-KT) and testosterone $(\mathrm{T})$ were low in females and gradually increased in the transitional and male phase. The present results suggest that low serum E2 levels and degeneration of oocytes accompanied by concomitant increase in the 11-KT levels and proliferation of spermatogenic germ cells are probably the events mediating protogynous sex change in E. merra (Figure 2) $[1,9]$. 


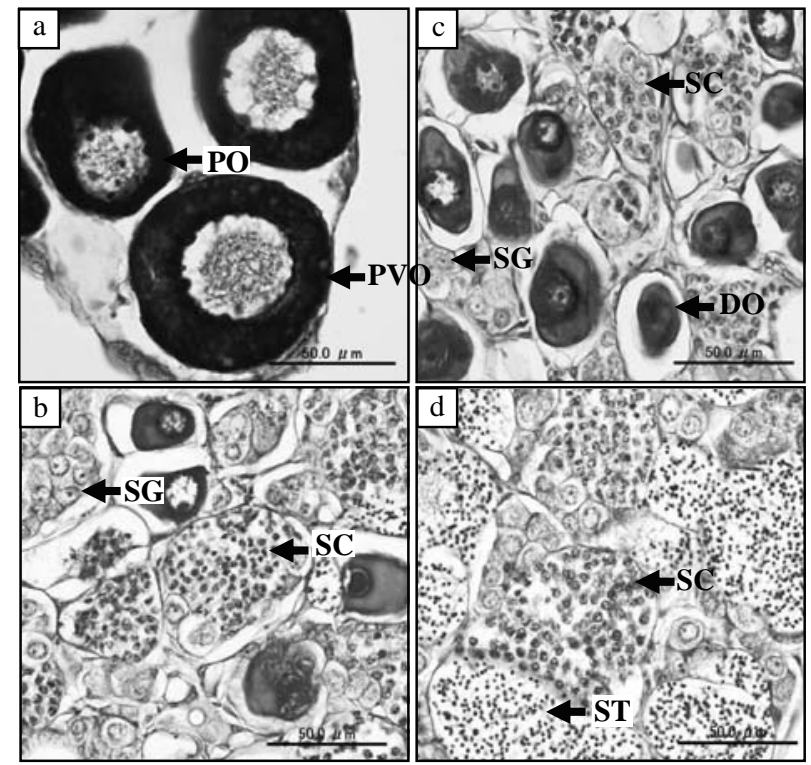

Fig. 1. Histological observation of the gonads during natural sex change in honeycomb grouper. (a) Female phase; (b) Early transitional phase; (c) Late transitional phase, and (d) Male phase.

Note: PO: primary oocyte; PVO: pre-vitellogenic oocyte; DO: degenerating oocyte; SG: sper-matogonia; SC: spermatocyte; ST: spermatid.

\section{The side of androgen production in the gonads}

In order to clarify a role of androgen play in sex change, we examined the dynamics of steroidogenic enzymes immunolocalization, viz cholesterol side-chain cleavage (450scc), biomarker of steroids and cytochrome

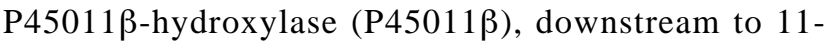
KT production, throughout the process of sex change in grouper. In female, P450scc immunoreactivity was observed in the thecal layer and tunica near blood vessels (BV) (Figure 3a, b). During the onset of sex change, P450scc reactive cells were observed in the remaining follicle layer of degenerated oocyte of the ovo-testis and tunica in early transitional (ET) and late transitional (LT) gonads. In male, P450scc was localized in the interstitial Leydig cells of testis. P45011 $\beta$ reactive cells were observed in the tunica near blood vessels in female but not in the theca layer (Figure 3c, d). In ET and LT phases gonads, P45011 $\beta$ appeared in remaining follicle layer of degenerated oocyte and tunica near BV. On the other hand, in male, both interstices and tunica near BV showed strong signals against $\mathrm{P} 45011 \beta$. Moreover, in vivo and in vitro levels of 11KT related with the changes in the nuclei diameter of $\mathrm{P} 45011 \beta$-positive cells in the both tunica near BV and remaining follicle layer of degenerated oocyte to interstices during the progress of sex change. The present results suggest that $11-\mathrm{KT}$ produced in the tunica near

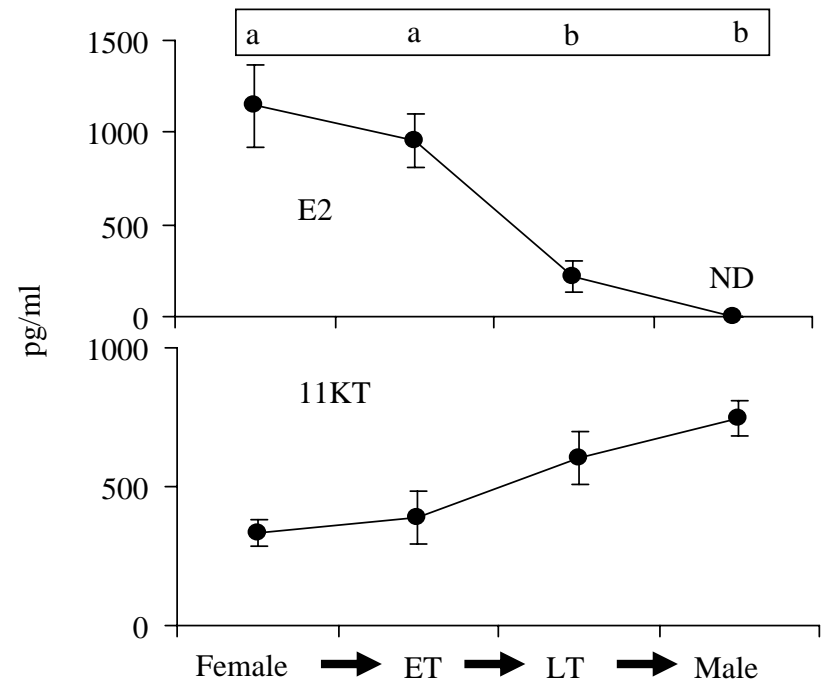

Fig. 2. Changes in plasma profile of $\mathrm{E} 2$ and 11-KT during the process of sex change.

Note: 1. Data are shown as the mean and SEM. Values not sharing a letter are significantly different by Tukey-Kramer HSD test (P $<0.05$ ).

2. ND: non-detectable; ET: Early transitional; LT: Late transitional.
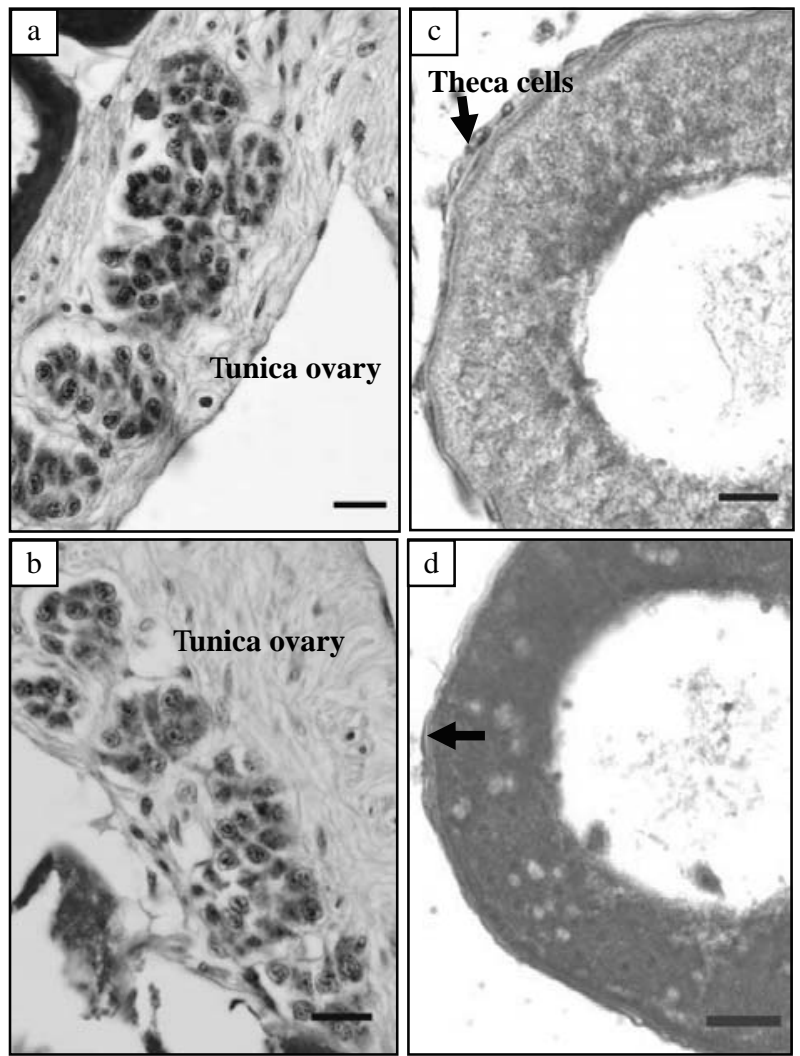

Fig. 3. Immunohistochemistry of P450sce and P45011 $\beta$ in gonads of E. merra. P450scc immunoreactivity in tunica and theca cells $(a, b) . P 45011 \beta$ like immunoreactivity only in tunica not in theca cells $(c, d)$. Arrowhead indicates positive reaction in theca cells (b). Scale bar $10 \mu \mathrm{m}$. 
BV may provide the stimulus for female to degenerate oocyte and initiate sex change. Moreover, 11-KT produced both in tunica near BV and remaining follicle layer of degenerated oocyte possibly play critical role during testicular differentiation as well as gonadal restructuring at mid to late phases of sex change $[1,3]$.

\section{Artificial sex change by the treatment of 11-ketotest- osterone}

To elucidate further the role of an androgen in sex change, we attempted to induce female to male sex change by exogenous $11-\mathrm{KT}$ treatments. The 75 days 11-KT treatment caused $100 \%$ masculinization of spawning females. Ovaries of the control fish had oocytes at various stages of oogenesis, while the gonads of the 11-KT treated fish had transformed into testes. In the sex-changed fish, plasma levels of E2 were significantly low, while both testosterone and 11-KT were significantly increased. Our results suggest that 11-KT plays an important role in sex change in the honeycomb grouper. Whether the mechanism of 11-KT induced female to male sex change acts through direct stimulation of spermatogenesis in the ovary or via the inhibition of estrogen synthesis remains to be clarified [5].

\section{Role of estrogen in sex change}

Circulating E2 levels decrease precipitously during female-to-male sex change. Whether this drop in E2 is a cause or consequence of sex change is still largely unknown. We treated adult female with aromatase inhibitor (AI, fadrozole), either alone or in combination with $\mathrm{E} 2$, to investigate the role of estrogen in protogynous sex change. Control fish had ovaries undergoing active vitellogenesis; the gonads of AI-treated fish had already developed into testes, which produced sperm capable of fertilization. In contrast, co-treatment of fish with E2 completely blocked AI-induced sex reversal. AI treatment significantly reduced circulating levels of E2, whereas the addition of E2 to AI prevented the loss. The plasma androgen ( $\mathrm{T}$ and 11-KT) levels were increased in the AI-treated fish, while the levels in the E2 supplemented fish were low compared to controls. Present results show that E2 plays an important role in maintaining female sex of hermaphrodite fishes, and that the inhibition of E2 synthesis causes oocyte degeneration leading to testicular differentiation in the ovary. A significant drop in endogenous E2 levels alone triggers female to male sex change, and the subsequent elevation of 11-KT levels correlates with progression of spermatogenesis $[6,7,8,11]$.

\section{Quickest sex change by aromatase inhibitor in the breeding season}

Artificial sex change was induced within two full moons by aromatase inhibitor during the breeding season to establish the quickest method of sex change and natural spawning of the honeycomb grouper. The sex change from female-to-male occurred during the time between the two full moons ( 3 weeks) following AI implantation in females just after spawning, and the efferent ducts of sex-changed males were filled with sperm (Figure $4 b$ ). In contrast all fish in control group had ovaries with matured oocytes (Figure 4a). To
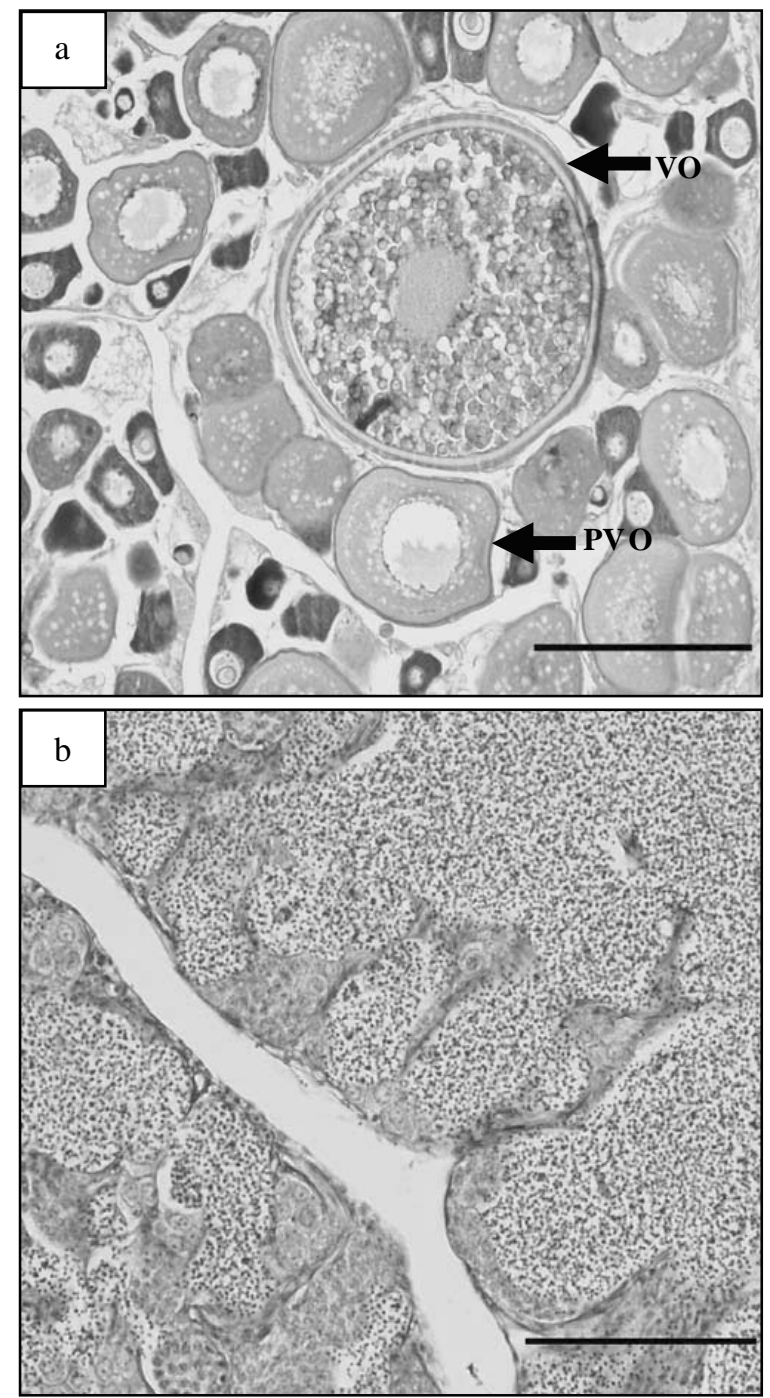

Fig. 4. The characteristics of gonadal histology in the sex change of E. merra by implantation with AI. (a) an ovary of control female mostly consisted with pre-vitellogenic oocyte (PVO) and vitellogenic oocyte (VO) and; (b) a testis of sex changed male filled with spermatozoa. Scale bar $50 \mu \mathrm{m}$. 


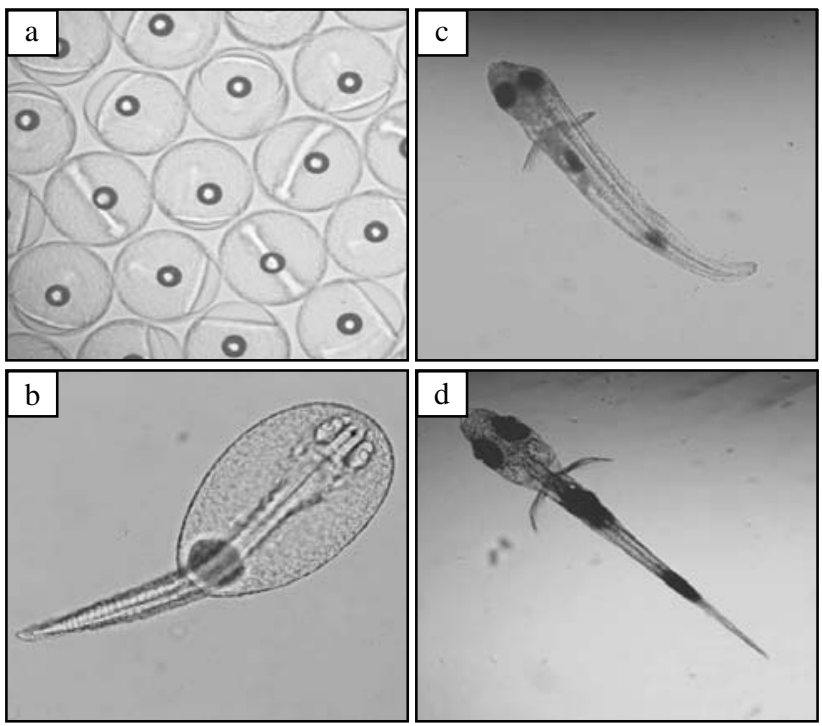

Fig. 5. Embronic and larval development of $E$. merra naturally spawned in the laboratory condition. (a) embryo after $7 \mathrm{hrs}$ of fertilization; (b) hatched larvae (1day after fertilization); (c) 2-day-old larvae; (d) 6-day-old larvae.

examine sperm fertility, sex-changed males were mated with natural, normal females and produced fertilized eggs (Figure 5a). Most of the hatched larvae grew normally without any morphological deformities (Figure $5 b, c, d)$. Therefore, the use of this method which is the quickest known sex change method using AI, may contribute to quality sperm production for grouper aquaculture [2].

\section{CONCLUSIONS}

The role of sex steroids in sex change is important both for commercial aquaculture development and for understanding the endocrine mechanisms of sex change. In E. merra, E2 and 11-KT are the predominant steroids regulating sex change. According to our current understanding, two possible manipulations are doable to initiate female-to-male sex change in protogynous grouper. One is the direct suppression of E2 production by blocking aromatase, thereby creating a suitable environment for the proliferation of gonial germ cells into spermatogonia, followed by spermatogenesis. Additionally, the use of exogenous $11-\mathrm{KT}$ can directly control oocyte degeneration or increase plasma androgen levels, allowing male germ cell proliferation, spermatogenesis, and sex change.

\section{ACKNOWLEDGMENTS}

These researches were supported by the Takeda Science Foundation and 21st Century COE project en- titled "The Comprehensive Analyses on Biodiversity in Coral Reef and Island Ecosystems in Asian and Pacific Regions" of the University of the Ryukyus, Okinawa, Japan, These studies were also supported by research project entitled "Utilizing advanced technologies in agriculture, forestry and fisheries", Japan and a Japanese Government (Monbukagaku-sho) Scholarship.

\section{REFERENCES}

1. Alam, M.A., Bhandari, R.K., Kobayashi, Y., Soyano, K., and Nakamura, M., "Changes in Androgen-Producing Cell Size and Circulating 11-Ketotestosterone Levels during Female-to-Male Sex Change in Honeycomb Grouper, Epinephelus merra," Molecular Reproduction and Development, Vol. 73, pp. 206-214 (2006).

2. Alam, M.A., Bhandari, R.K., Kobayashi, Y., Soyano, K., and Nakamura, M., "Induction of Sex Change Within Two Full Moons during Breeding Season and Spawning in Grouper," Aquaculture, Vol. 255, pp. 532-535 (2006).

3. Alam, M.A., Komuro, H., Bhandari, R.K., Nakamura, S., Soyano, K., and Nakamura, M., "Immunohistochemical Evidence Identifying the Site of Androgen Production in the Ovary of the Protogynous Grouper, Epinephelus merra," Cell and Tissue Research, Vol. 320, pp. 323-329 (2005).

4. Allsop, D.J. and West, S.A., "Sex Change Life History Invariants in Fish," Journal of Evolutionary Biology, Vol. 16, pp. 921-929 (2003).

5. Bhandari, R.K., Alam, M.A., and Nakamura, M., "Induction of Female-to-Male Sex Reversal in the Honeycomb Grouper, Epinephelus merra, by 11-Ketotestosterone Treatments," Zoological Science, Vol. 23, pp. 65-69 (2006).

6. Bhandari, R.K., Alam, M.A., Higa, M., Soyano, K., and Nakamura, M., "Evidence That Estrogen Regulates the Sex Change of Honeycomb Grouper, Epinephelus merra, a Protogynous Hermaphrodite Fish," Journal of Experimental Zoology, Vol. 330A, pp. 497-503 (2005).

7. Bhandari, R.K., Higa, M., Nakamura, S., and Nakamura, M., "Aromatase Inhibitor Induces Complete Sex Change in a Protogynous Honeycomb Grouper, Epinephelus merra," Molecular Reproduction and Development, Vol. 67, pp. 303-307 (2004).

8. Bhandari, R.K., Komuro, H., Higa, M., and Nakamura, M., "Sex Inversion of Sexually Immature Honeycomb Grouper, Epinephelus merra, by aromatase inhibitor," Zoological Science, Vol. 21, pp. 305-310 (2004).

9. Bhandari, R.K., Komuro, H., Nakamura, S., Higa, M., and Nakamura, M., "Gonadal Restructuring and Correlative Steroid Hormone Profiles during Natural Sex Change in Protogynous Honeycomb Grouper, Epinephelus merra," Zoological Science, Vol. 20, pp. 1399-1404 (2003). 
10. Frisch, A., "Sex-Change and Gonadal Steroids in Sequentially-Hermaphroditic Teleost Fish," Review in Fish Biology and Fisheries, Vol. 14, pp. 481-499 (2004).

11. Nakamura, M., Kobayashi, Y., Miura, S., Alam, M.A., and Bhandari, R.K., "Sex Change in Reef Fishes," Fish Physiology and Biochemistry, Vol. 31, pp.117-122
(2006)

12. Perry, M.N. and Grober, M.S., "A Model for Social Control of Sex Change: Interactions of Behavior, Neuropeptides, Glucocorticoids, and Sex Steroids," Hormones and Behavior, Vol. 43, pp. 31-38 (2003). 